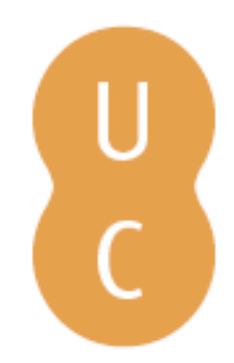

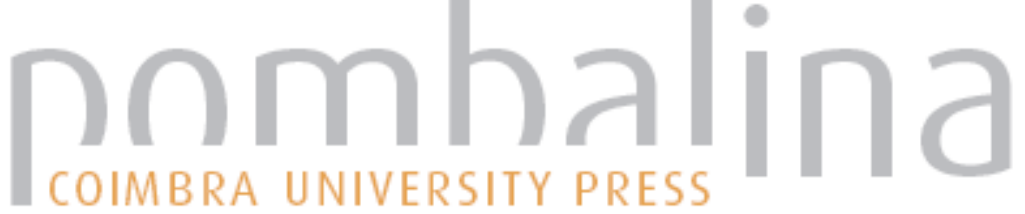

\section{La táctica en el tenis de individuales}

Autor(es): $\quad$ Sanz, David; Pedro Fuentes, Juan; Crespo, Miguel

Publicado por: Imprensa da Universidade de Coimbra

URL

persistente: URI:http://hdl.handle.net/10316.2/41771

DOI: $\quad$ DOl:https://doi.org/10.14195/978-989-26-1286-7_10

Accessed : $\quad$ 26-Apr-2023 11:44:46

A navegação consulta e descarregamento dos títulos inseridos nas Bibliotecas Digitais UC Digitalis, UC Pombalina e UC Impactum, pressupõem a aceitação plena e sem reservas dos Termos e Condições de Uso destas Bibliotecas Digitais, disponíveis em https://digitalis.uc.pt/pt-pt/termos.

Conforme exposto nos referidos Termos e Condições de Uso, o descarregamento de títulos de acesso restrito requer uma licença válida de autorização devendo o utilizador aceder ao(s) documento(s) a partir de um endereço de IP da instituição detentora da supramencionada licença.

Ao utilizador é apenas permitido o descarregamento para uso pessoal, pelo que o emprego do(s) título(s) descarregado(s) para outro fim, designadamente comercial, carece de autorização do respetivo autor ou editor da obra.

Na medida em que todas as obras da UC Digitalis se encontram protegidas pelo Código do Direito de Autor e Direitos Conexos e demais legislação aplicável, toda a cópia, parcial ou total, deste documento, nos casos em que é legalmente admitida, deverá conter ou fazer-se acompanhar por este aviso. 
IMPRENSA DA UNIVERSIDADE DE COIMBRA

COIMBRA UNIVERSITY PRESS

RAUL A.

MARTINS

GONÇALO DIAS

PEDRO CABRAL MENDES

\section{EDITORES}

ESTRATÉGIA

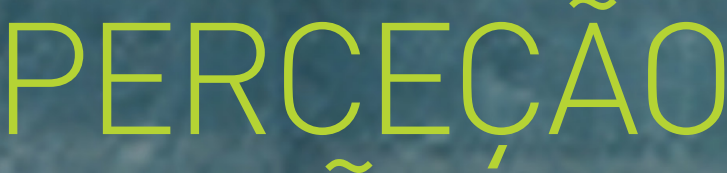

EAGÃA

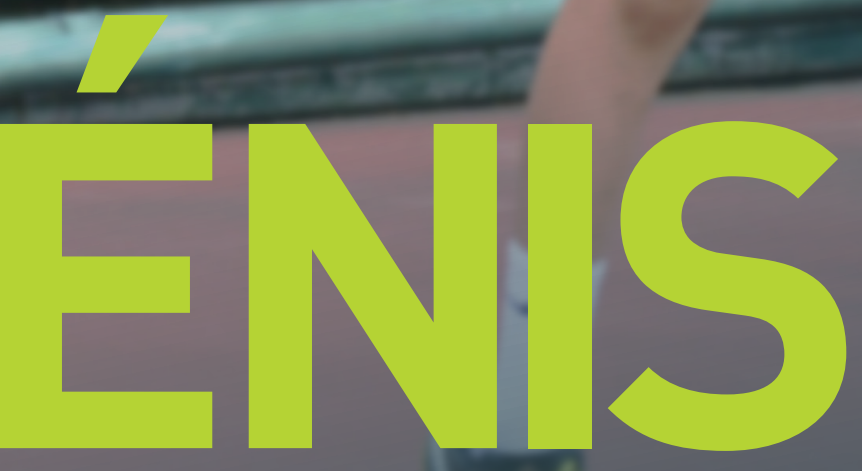




\section{CA P ÍTULO 10}

\section{LA TÁC TICA E N EL T ENIS DE INDIVIDUALES}

David Sanz

Juan Pedro Fuentes

Miguel Crespo

\section{Introducción}

El tenis es un deporte considerado complejo desde el punto de vista de su estructura y desarrollo del propio juego. Durante el mismo se dan fases variables por cuanto se refiere a los tiempos de juego, número de golpes por punto, velocidad del juego, requerimientos de tomas de decisiones con mayor y menor incertidumbre, marcador que condiciona constituyéndose en un elemento que añade un mayor estrés a la toma de decisiones y a la propia ejecución, la propia incertidumbre que existe en cada golpeo que realiza el rival. Por todos estos factores, podemos considerar que este juego es dinámico, complejo y con un alto grado de incertidumbre, lo que le aporta unas características muy concretas y le diferencia de otras modalidades deportivas.

En este capítulo nos centraremos en la táctica, concretamente en la de individuales y, en línea con el planteamiento actual en el deporte por lo que respecta al entrenamiento, en metodologías para su desarrollo e incluso el propio análisis del juego, donde se sugiere que la forma de abordarlo debería ser un planteamiento mucho más 
holístico e integral, ya que es difícil en deportes complejos atomizar los diferentes componentes y estudiarlos por separado, puesto que todos ellos tienen una incidencia y compromiso directo sobre el resto. Así, por ejemplo, desde el punto de vista técnico podemos analizar una derecha y describir el patrón mecánico de golpeo, si bien es cierto, que sacada del contexto de juego en el que se produce, podemos estar obviando factores como el marcador, que potencialmente condicionan las acciones técnicas. De ahí que propongamos modelos más integradores, donde se vinculen de forma indisociable la técnica y la táctica, es decir las acciones con las intenciones, atendiendo a las interacciones que tienen los movimientos y acciones con el medio y la situación, en línea con lo que proponen algunas líneas de investigación como la aplicación de teorías y conceptos ecológicos, que relacionan la toma de decisiones con principios dinámicos y en donde las posibilidades de acción se perciben en función de los datos ambientales (Araújo, 2005; Araújo et al., 2006)

En este sentido, entendemos la táctica en el tenis como las acciones que se desarrollan basadas en la toma de decisión del jugador, permitiendo resolver la situación problema que supone cada golpeo de pelota durante el juego. De esta forma, la táctica en el tenis supone una resolución instantánea ante la pelota que me viene del contrario y, por ende, está vinculada estrechamente con los tres principales estímulos que compone el juego del tenis, el adversario, la pelota y el espacio de juego: El adversario, entendido como el jugador de referencia que condiciona en cada momento la toma de decisiones a realizar, bien sea por la acción que desarrolle en un golpeo concreto o, simplemente, por la ubicación espacial que disponga durante el juego; la pelota, por las características de la misma respecto a la calidad del golpeo del adversario, donde la altura, velocidad, cantidad de efecto condicionarán toma de decisiones en cada momento; y, por último, el espacio de juego, ya no tanto desde el punto de vista de la superficie, que obviamente 
supone un condicionante, sino desde el punto de vista de la generación de espacios que se va creando durante el intercambio en oposición de los dos jugadores y que constantemente tengo que ir analizando teniendo en cuenta el espacio donde me muevo (espacio propio) y el espacio donde envío la pelota - espacio adverso (Sanz et al., 2004).

El concepto de estrategia, asociado de forma directa a la táctica, supone un proceso de preparación y estudio de la situación a la que se va enfrentar el jugador, analizando los componentes que le permitan establecer una intervención potencialmente exitosa frente a su adversario. En este estudio y diseño de la estrategia se tendrán en cuenta algunos componentes tales como los puntos fuertes y débiles del adversario, la superficie de juego, las condiciones climatológicas, los encuentros previos disputados con el jugador,..., entre otros. Así, tal y como indican Over y O'donoghue (2010), estrategia y táctica son conceptos relacionados en el rendimiento deportivo, donde la estrategia se planifica antes de la competición para utilizar de la mejor manera posible las fortalezas del jugador, limitando los efectos de cualquier debilidad. Al mismo tiempo, la estrategia debe tratar de explotar las debilidades conocidas del adversario, evitando que pueda hacer uso de sus fortalezas.

En este sentido, a la hora de categorizar la acción táctica podríamos hablar de una serie de principios que enmarcan la misma (adaptado de Riera, 2003 y Sanz, 2015), tales como:

- Subordinación - donde la estrategia prevista deberá estar subordinada a la táctica en la situación real de juego.

- Imprevisibilidad - dado el carácter abierto de nuestro deporte donde cualquier situación puede ser totalmente diferente de la anterior y de la posterior.

- Interdependencia - puesto que la táctica ha de reflejar la influencia del adversario, de la situación, del marcador.... 
- Adaptabilidad - a lo largo de la evolución del partido donde nos tendremos que ir amoldando a las situaciones que se vayan sucediendo.

- Validez - a través de indicadores que me permitan realmente obtener información veraz de lo que está ocurriendo.

- Temporalidad - a través de la cual podremos acotar las acciones y describir un perfil comportamental de nuestro adversario.

- Intencionalidad - puesto que las actuaciones llevadas a cabo tendrán como sustrato el objetivo que se pretende conseguir.

- Arbitrariedad - donde el jugador, constantemente puede ir cambiando su estrategia y, por lo tanto, en función de la situación de juego en la que se encuentre, él mismo tienen la capacidad y posibilidad de ir modificando no sólo la estrategia establecida, sino también la táctica durante el desarrollo del juego.

- Objetividad - puesto que pese al propio desarrollo del juego, el proceso de análisis táctico que realice el jugador y entrenador y que condicionará las posteriores tomas de decisión, deberá realizarse de una forma lo más objetiva y "aséptica" posible, evitando que las emociones no controladas por las acciones previas, precipiten la toma de decisiones.

La táctica en el tenis de competición y alta competición supone uno de los componentes del juego más relevantes en el rendimiento de los jugadores, de hecho, tal y como afirman Crespo y Reid (2002), los jugadores y entrenadores afirman que las mejoras que se realicen en cualquier destreza estratégica y táctica serán también decisivas para la evolución óptima del tenista.

Por esta razón, para los entrenadores, la táctica se convierte en uno de los componentes del juego que más presentes deben tener en sus entrenamientos, ya que, tal y como apuntan, es el factor que marca la diferencia entre los buenos jugadores y los mejores. Incluso algunos entrenadores de alto nivel, como Javier Piles, coach 
de David Ferrer durante gran parte de su carrera deportiva, señalaba que la diferencia de los jugadores en el ranking se basaba en que los jugadores mejores clasificados eran aquellos que cubrían los tres parámetros exigibles en el tenis de alta competición: Patrón de juego+Velocidad de piernas+Velocidad mental en la toma de decisiones. (Piles y Crespo, 2012). De hecho, la evolución del propio juego por lo que respecta a los materiales, superficies, condición física de los jugadores, entre otros, ha permitido que haya una notable evolución de la táctica desde hace unos años hasta la actualidad y pese a que las situaciones de juego son las mismas (tipificadas como servicio, resto, fondo-fondo, red y fondo-red), los estilos de juego a lo largo del tiempo han ido variando y, de hecho, se han ido homogeneizando, de forma que ya no hay patrones tácticos tan diferentes y marcados entre los jugadores, tanto en el tenis femenino como en el masculino. En este sentido, en el tenis profesional podemos percibir patrones de juego mucho más definidos y estables que en el tenis de categorías junior o en la etapa de transición de junior a profesional. Esto no quiere decir que en los proceso de formación tengamos que limitar el aprendizaje y entrenamiento de los diferentes estilos de juego que nos permiten las situaciones de juego en el tenis, pero si es cierto que a medida que el jugador vaya evolucionando, deberemos ir perfilando y seleccionando que estilo se adapta mejor a sus características para aportarle ese patrón táctico que le permita la seguridad y confianza de poder recurrir al mismo para poder actuar y reaccionar rápidamente, tal y como exige el tenis en el alto nivel (Piles y Crespo, 2012).

Así, algunas de las características que podemos destacar dentro de lo que conocemos como la táctica moderna serian (Crespo y Reid, 2002):

- La potencia se usa como un arma táctica: La potencia se ha ido convirtiendo en el elemento distintivo del juego. El desarrollo 
físico de los jugadores y el somatotipo de los mismos, junto con la evolución de los materiales hace que hoy en día la potencia de los golpeos y las velocidades que alcanza la pelota sean mucho mayores. En este sentido, golpeos como el resto y el servicio se han convertido en unos claros indicadores de rendimiento y de predicción de éxito en un partido (Filipcic et al., 2015).

- La toma de decisiones es más rápida: los tenistas tienen cada vez menos tiempo para decidir que golpe y dirección le darán a la pelota.

- El uso de los mejores golpes: la mayoría de jugadores tiene la derecha y el servicio como los dos golpes en los que basa su juego, aunque uno de los golpes que ha sufrido una gran evolución, gracias al apoyo de la doble mano sobre la empuñadura, es el revés.

- La carencia de especialistas en estilos de juego específicos: hoy en día el circuito profesional es más homogéneo, ya que se disputa gran parte de la competición en superficie rápida no porosa y esta situación junto con la adaptación constante que los jugadores tienen que hacer a lo largo del año para disputar el circuito y los Grand Slams, hace que se hayan convertido en jugadores más "completos", capaces de jugar y adaptarse rápidamente de tierra a hierba y/o a superficie rápida de resinas, manteniendo un estilo de juego similar que hace que los "especialistas" como existía antaño de "servicio-red", "jugador de tierra"..., hayan dejado paso a este "jugador polivalente", que mantiene prácticamente la misma estructura de juego en todas las superficies.

Finalmente, para concluir este primer apartado del capítulo, consideramos interesante exponer algunos conceptos tácticos relacionados con el rendimiento en el tenis y que algunos entrenadores sugieren como exitosos. En este sentido, partiendo 
de la propuesta de Tiley (2002), en la que se plantean algunos conceptos a tener en cuenta en el tenis de individuales, sugerimos algunas consideraciones al respecto:

- Jugar con porcentajes consistentes, es decir, evitar fallos bien sean forzados o en situaciones no forzadas, aumentando el número de puntos conseguidos por forzar el fallo del oponente. En el tenis de individuales los 3 golpes más importantes para la consistencia son el servicio, la devolución y la subida a la red.

- Conocer las zonas: de forma que el jugador, atendiendo al espacio propio, sea capaz de jugar acorde a la situación espacial. Aquí se utiliza la analogía del semáforo, donde la Zona Roja es la zona cercana a la línea de fondo en la que normalmente se mantendrá una actitud neutra o defensiva; la Zona Naranja es la zona de tres cuartos de pista, donde el jugador deberá intentar forzar la situación para comprometer al rival; y, finalmente, la Zona Verde, entendida como la zona cercana o en los propios cuadros de servicio, en donde el jugador debe ganar el punto y mantener una actitud netamente ofensiva.

Nosotros utilizamos también esta analogía con los mismos conceptos pero atendiendo al tipo de pelota al que se enfrenta el jugador, por lo que no solamente hablamos de un concepto espacial, sino del tipo y calidad de pelota a la que te enfrentas. De esta forma, una pelota en Zona Roja que normalmente sería defensiva, para un jugador de nivel, si la pelota no viene con potencia, podría ser perfectamente un golpeo que corresponda a un tiro ganador o que fuerce la posición del adversario, es decir un tiro que correspondería más con la Zona Naranja o Verde.

- Comprender las Zonas-Objetivo, atendiendo a la disposición espacial en la que se encuentre el jugador, así por ejemplo 
desde posiciones alejadas los tiros tendrán un mayor componente de parábola y profundidad, con un margen de seguridad por encima de la red. Las posiciones cercanas a la red permitirán abrir más ángulos y tiros más bajos. Márgenes de seguridad en la pista, evitando las zonas excesivamente cercanas a las líneas.

- Limitar los cambios de dirección, dado que la posibilidad de cometer un error aumenta cuando se cambia la dirección de entrada de la pelota.

- Centrar la pelota, evitando jugar demasiado largo y provocando que el adversario disponga de menos zonas de juego y no pueda abrir ángulos.

- Atacar la pelota corta, de forma que se pueda tomar la iniciativa en el punto atacando el lado más débil del rival y consiguiendo acceder a la red. La eficacia en la red supone ganar dos de cada tres puntos $(66 \%)$.

- Secuencia 1-2, manteniendo una actitud ofensiva de forma que el primer tiro fuerce y el segundo sea el que consiga el punto, como por ejemplo a través de un passing indirecto cuando el adversario sube a la red, donde la primera pelota le forzamos para que volee incómodo y en la segunda buscamos el tiro ganador.

- Cambia el ritmo, de forma que el oponente no tenga muchas opciones de estar cómodo en su juego, por lo que debemos cambiar el ritmo de juego a través del tipo de golpes, los efectos, las alturas, la velocidad..., aportando la mayor incertidumbre posible al adversario siempre que sea posible.

- Jugar con márgenes, de forma que si estamos defendiendo enviemos nuestros tiros alejados de las líneas limítrofes de la pista (individual y fondo), mientras que si estamos atacando de veremos forzar la situación, con envíos cercanos a las líneas.

- Desplazar al oponente, consiguiendo que llegue incómodo a la pelota y que evite situaciones estables de golpeo, por lo que 
los cambios de dirección y los contrapiés serán clave a la hora de generar espacios libres en la pista.

Respecto al tenis femenino, pese a que los conceptos anteriores los podemos aplicar perfectamente, es cierto que se mantienen algunas diferencias respecto a los patrones tácticos que se siguen en el tenis individual masculino. De hecho, a finales del siglo $\mathrm{XX}$ podemos hablar del comienzo de una etapa en la que se va adquiriendo un patrón de juego dominante basado en la fuerza y la velocidad de los golpes más que en los aspectos tácticos y la comprensión del juego (Van Aken, 2002). Así, podemos confirmar que hoy en día el tenis femenino profesional es más rápido, los intercambios de puntos son más cortos y mucho más intensos (Martens y Maes, 2005). En la misma línea, Antoun (2007) señala que hoy en día en el tenis femenino existe menos variedad que antes porque las jugadoras golpean más fuerte, más plano y desde una posición más agresiva en la pista sin emplear mucho efecto. En este sentido, el debate se genera en si jugar con mayor variedad podría ser un hecho diferenciador e incluso un factor que predisponga a la obtención de mejores resultados, como sugiere el propio Antoun (2007) y Rodriguez (2012), incorporando golpeos como el revés cortado agresivo y el liftado con ángulo corto, el golpe de recuperación liftado, la dejada, el globo agresivo, la volea por sorpresa, la volea liftada y el golpe de fondo alto de ataque, en suma, una serie de acciones que aporten un mayor repertorio y mayor versatilidad en los patrones tácticos.

\section{Modelos de análisis táctico}

Una vez introducidos los conceptos básicos sobre la táctica y las orientaciones básicas al respecto de la misma, pasaremos a otro de los apartados que más se ha venido trabajando en los últimos años, 
y que consiste en el estudio de la táctica para poder extraer conclusiones que nos permitan orientar, asesorar y diseñar entrenamientos para nuestros jugadores con la intención de la mejora del rendimiento.

El análisis de la acción táctica en el juego del tenis ha sido objeto de investigación desde hace más de 20 años, si bien es cierto que en los últimos años ha sufrido un notable avance y desarrollo, en consonancia con lo que ocurre en otros deportes respecto del seguimiento de jugadores en la acción de juego para extraer indicadores que permitan orientar las futuras intervenciones, ayudado por el incremento de nuevas tecnologías que permiten mucha mayor precisión, fiabilidad, portabilidad de los equipos de medida y, sobretodo, inmediatez en los datos obtenidos y en la interpretación de los mismos. Autores como Reid (2011) apuntaba que la figura del "socuter" en el tenis será uno de los componentes de los equipos en jugadores profesionales y, de hecho, aunque no se hace de forma pública, conocemos diferentes jugadores profesionales que ya han incorporado actualmente este profesional en sus equipos.

Hoy en día existen dos modelos básicos de análisis en el estudio de la acción táctica, los modelos de seguimiento o "tracking" y los modelos de etiquetado o "tagging". Los primeros se basan en el seguimiento de los jugadores a través de sistemas tecnológicos que permiten identificar el jugador, la pelota y el espacio, y posteriormente analizar todas las variables que se puedan derivar de estos componentes. Los segundos, más asequibles y habitualmente los más utilizados, se basan en la identificación de una serie de indicadores que se registran a través de unas etiquetas diseñadas de forma previa, de forma que se recogen y posteriormente se pueden analizar tanto visualmente como estadísticamente.

La mayoría de los modelos de análisis se basan en la observación como principal instrumento de medida. Esta observación sistemática es un proceso que nos proporcionará información medible y además cuantificable sobre el hecho que estemos evaluando. 
Tal y como señalan Sanz y Terroba (2012), la observación del técnico se ha basado habitualmente en la visión del entrenador respecto la acción objeto de estudio, es decir, en el "ojo clínico" del entrenador. Este sistema tiene un valor intrínseco muy importante dado el grado de conocimiento que tienen los técnicos respecto a las situaciones a evaluar y analizar, pero, sin embargo, cuando hablamos de precisión en la evaluación, las propias limitaciones sensoriales de nuestros sistemas de captación de información hacen que puedan quedar lagunas en esos procesos de observación y que, por lo tanto, la intervención sobre los mismos pueda estar sesgada, condicionada y no ser tan precisa como cuando llevamos a cabo una observación rigurosa, sistemática y objetiva. En el ámbito de la investigación, será la metodología observacional la que sentará las bases para el diseño de trabajos de investigación centrados en estos análisis (Anguera, 1990; Anguera et al., 2000). Así empezaron las primeras tesis doctorales en tenis publicadas sobre el análisis táctico del tenis utilizando la metodología observacional (Gorospe, 1999; Gorospe et al., 2005; Garay, 2003).

Pasaremos a continuación a exponer la evolución que se ha ido dando en los diferentes modelos de análisis de los patrones tácticos en el tenis a los largo de estos últimos 20 años, en los que se han empleado básicamente cuatro modelos de análisis, al que añadimos un último modelo que ofrece un cambio de paradigma respecto algunos de los análisis señalados anteriormente (Sanz y Terroba, 2012):

- Empleo de sistemas de registro manual, desde aquellos donde el técnico anotaba en una libreta un registro de acontecimientos de los sucedido respecto a aspectos tales como puntos fuertes, débiles, movilidad, comportamiento ante situaciones complejas..., empleando una planilla de registro estructurada y que posteriormente han ido evolucionando, gracias a la 
tecnología a PDAs o a Smartphones que cuentan con aplicaciones que permiten registrar las variables anteriormente señaladas. Obviamente estos sistemas son útiles, pero permiten recoger solo datos globales, la mayoría vienen prediseñados respecto a las variables que se pueden recoger y no es posible asociar las imágenes de forma directa a los registros.

- Empleo de sistemas de captura de vídeo, utilizados fundamentalmente para el análisis de acciones técnicas, han dejado paso al análisis de las secuencias de acciones y a poder asociar las imágenes de vídeo a la toma de decisiones de los jugadores a lo largo de un partido. Esto es posible gracias a los softwares que vinculan, mediante el etiquetado, los momentos o situaciones de un partido para luego poder reproducirlos y analizarlos una vez categorizados. Este proceso no se hace de forma automática por el momento y se debe realizar de forma manual, pese a que ya han comenzado a aparecer algunas alternativas totalmente automáticas (Almajai et al., 2010; Christmas et al., 2005) e incluso, aprovechando la tecnología de recogida de imágenes mediante cámaras como Hawk Eye (www.hawkeyeinnovations.com) o recientemente sistemas como Playsight (www.playsight.com), de forma automática y durante el propio juego, la disposición de una serie de cámaras nos permite obtener datos cinemáticos tales como velocidades medias de los jugadores, aceleraciones, distancias recorridos,.. así como otras variables de componente más táctico tales como análisis de golpeos (\% de $1^{\circ}$ y $2^{\circ}$ servicios, restos), número de golpes por punto/partido, winners, errores, etc., y todo esto sin la necesidad de que el jugador deba llevar un sensor.

- Empleo de análisis estadísticos, al objeto de intentar conseguir la mayor información posible y procesarla para poder conseguir una rápida interpretación de los datos a partir de 
la potencia estadística de los análisis realizados. Goldstein (1976) ya afirmaba la importancia que un buen análisis estadístico de un partido puede tener con el objetivo proporcionar casi todas las respuestas sobre los puntos jugados durante el partido. De esta forma se han ido sucediendo una serie de trabajos como por ejemplo el modelo de cadena de Markov establecido por Schutz (1970), con una probabilidad constante de ganar el punto. El programa informático de Klaassen y Magnus (2003) denominado TENNIS PROB, el cual calculaba de forma rápida y automática las probabilidades de victoria en un partido, así como otros estudios en esta línea de análisis estadísticos y probabilísticos como los de Gale (1971), George (1973), Norman (1985), Croucher (1986), Pollard (1983), Barnett y Clarke (2002, 2005), Barnett (2005), o Barnett y colaboradores (2006), entre otros.

- Empleo de minería de datos e inteligencia artificial, que pese a ser un tipo de aplicación más propia de otras disciplinas y áreas de conocimiento, como en el ámbito del marketing, la salud, la empresa, se ha ido incorporando progresivamente en el ámbito deportivo, con el mismo propósito a los anteriormente citados, el de encontrar patrones y posibles relaciones en los datos que puedan resultar en nuevos conocimientos, pudiendo las mismas predecir futuros resultados. Los objetivos principales que se plantean normalmente son: scouting de nuevos jugadores, predicciones de resultados y medición de rendimiento. Sin embargo, existen relativamente pocos estudios donde se emplea la minería de datos para extraer patrones tácticos (Terroba et al., 2010; Vis et al., 2010). Por otro lado, el empleo de técnicas de aprendizaje por refuerzo, redes neuronales, teoría de los sistemas dinámicos (Palut \& Zannone, 2005) y teoría de juegos aplicadas al análisis deportivo no ha hecho sino comenzar. 
- Propuesta de cambio de paradigma, a tenor de los estudios realizados en los diferentes soportes anteriormente comentados, Sanz y Terroba (2012) plantearon la importancia de establecer los criterios clave sobre los que queramos realizar un análisis. En la línea de lo que algunos autores señalan respecto a qué información de la que podemos extraer es realmente interesante y útil (Barnett \& Clarke, 2005; Barnett et al., 2008; Gillet et al. 2009; O`Donoghue, 2001; Pollard et al., 2010; Reid et al., 2010; Over \& O’Donoghue, 2008, 2010).

Desde este punto de vista, alejados de los modelos de análisis finalistas sobre el último golpe en cada punto, se propone un estudio a partir de los momentos donde el jugador consigue generar una situación de desequilibrio, puesto que ese será el momento clave de análisis para ver cómo se desarrolla la acción posterior y la anterior, si queremos establecer cadenas secuenciales basadas en un criterio objetivo que se aleje del error forzado y no forzado que no viene vinculado a qué acción ha provocado esa situación y, sobre todo, como categorizamos el no forzado, si puede ser una situación de 15-40 para hacer un break y si estamos al servicio, esa situación, pese a lo sencilla que parezca, el error no podría categorizarse como erro no forzado.

\section{Resultados de las investigaciones en la última década}

Al objeto de complementar este apartado sobre los modelos de análisis de la táctica que se han utilizado en el tenis, proponemos una revisión de las investigaciones más relevantes hasta la fecha en este ámbito, a partir de la revisión realizada por Crespo y Sanz (2011) y centrándonos en los últimos diez años. Pasaremos a exponer los diferentes estudios basados en 6 categorías: 
1. Estadísticas sobre golpes

2. Datos sobre probabilidad de victoria y patrones de juego tácticos

3. Diferencias entre el tenis masculino y femenino

4. Superficie y estrategia

5. Hipótesis sobre táctica y estrategia en el tenis

6. Importancia de los puntos, juegos y sets en tenis

\section{Estadísticas sobre golpes}

Respecto al golpe de servicio, tal y como se ha concluido en diferentes investigaciones (Barnett et al., 2008; Pollard et al., 2009; Bedford et al., 2009), la utilización de determinadas estrategias en el servicio mejora el rendimiento del jugador de tenis de alto nivel.

Klaassen y Magnus (2007) en el torneo de Wimbledon observaron que cuando las pelotas están más usadas aumentaba la probabilidad de meter el primer servicio. Por su parte, Pollard y Barnett (2007) demostraron que, según sus datos, existe una ventaja psicológica cuando se saca primero en el set. Para Magnus y Klaassen (2007) es conveniente elegir primero sacar debido a que estadísticamente se dan menos rupturas de servicio en el primer juego del partido que más adelante. La razón es porque en el primer juego, el restador aún no está restando al más alto nivel y por ello, si se gana el sorteo, es mejor elegir sacar.

Newton y Keller (2005) estudiaron la probabilidad de ganar un juego, set y partido basándose en la probabilidad de cada jugador en ganar el punto con su servicio, que asumieron que eran variables aleatorias independientes e idénticamente distribuidas. Los autores concluyeron que la probabilidad de ganar un set y un partido es independiente de quien fuera el jugador que sacara primero.

Magnus y Klaassen (2007) en su estudio con datos de partidos de Wimbledon midieron la calidad del segundo servicio considerando 
el porcentaje de puntos ganados con el segundo servicio y llegaron a la conclusión de que el dicho: "un jugador es tan bueno como su segundo servicio" no es cierto. Lo cierto es que "un jugador es tan bueno como su primer servicio". Para estos autores, el primer servicio es más importante que el segundo.

Por otro lado, Pollard y Pollard (2007) analizaron la situación en la que el sacador podría arriesgarse constantemente en su servicio. Llegaron a la conclusión de que lo mejor era que el sacador sacara el primer saque con mayor riesgo y el segundo saque con menor riesgo.

Barnett y colaboradores (2008) analizaron la posibilidad de que los sacadores optaran por realizar dos saques potentes según el tipo de la superficie de la cancha, así como las características del servicio y la devolución de ambos jugadores. Dichos autores compararon las estrategias de servicio entre Nadal y Roddick y concluyeron que este último podría cambiar su estrategia de servicio cuando jugara en hierba contra Nadal utilizando dos primeros servicios en lugar de un primero y un segundo más lento o bien combinando un saque más fuerte y otro más débil cuando sacara su segundo saque contra Nadal.

Por su parte, Pollard (2008) analizó la típica situación en la que un saque de riesgo medio tiene un resultado cuadrático en lugar de lineal, que da más peso al resultado de sacar con alto riesgo que con bajo. Pollard y colegas (2009) modelaron la situación para permitir la posibilidad de que los jugadores cambiaran sus estrategias de saque durante el transcurso del partido. Bedford y colaboradores (2010) demostraron que los sacadores tienen que tomar más riesgo en los puntos más "importantes" y menos riesgo en los puntos menos "importantes".

Pero, a pesar de que hay una gran cantidad de estudios sobre estrategias de servicio en el tenis, muchas de ellas no han sido presentadas de manera que sea posible utilizar sus conclusiones de forma práctica por parte de entrenadores y científicos del deporte para mejorar potencialmente el rendimiento de los tenistas. Además, algunos de los modelos de las investigaciones antes presentadas 
no calculan de forma precisa el alto o bajo riesgo del sacador durante el partido debido a la falta de disponibilidad de datos. Esta situación es posible calcularla ahora debido a la disponibilidad de paquetes informáticos de tenis que capturan las estadísticas del partido en la mayoría de los torneos de alto nivel y permiten a los investigadores tener acceso directo a estos datos.

Otros estudios que analizaron los golpes de servicio y resto fueron los de Gillet y colaboradores (2009), realizado sobre partidos de Roland Garros en los años 2005 y 2006 con el objetivo de establecer patrones de juego y estrategias, además de determinar la influencia de estos golpes en el resultado final del punto. Comprobaron que el porcentaje de puntos ganados con servicio plano, concretamente el dirigido a la zona de la "T", fue mayor que el porcentaje de puntos ganados ejecutando servicios con efecto liftado y cortado. En cuanto a los restos, cuando los jugadores los dirigieron a la zona central de la pista obtuvieron un mayor porcentaje de puntos ganados que cuando el resto iba dirigido a zonas laterales de la pista. De igual forma, el estudio de Hizan y colegas (2011) con jugadores sub 16 y sub 12, en el que comprobaron que los jugadores profesionales consiguieron un mayor número de servicios directos que los jugadores sub 16 y sub 12 y que las jugadoras profesionales. Éstas a su vez consiguieron más servicios directos que las jugadoras sub 12 . Independientemente del género, los jugadores profesionales cometieron menos dobles faltas y consiguieron un mayor porcentaje de puntos ganados restando segundo servicio que los jugadores sub 16 y sub 12 (Martínez-Gallego, 2015).

\section{Datos sobre probabilidad de victoria y patrones de juego tácticos}

Barnett y Taylor (2005) estudiaron la predicción de resultados de partidos en el Abierto de Australia y utilizaron las estadísticas 
habituales publicadas por la ATP para realizar dichas predicciones y para actualizarlas durante el transcurso del partido. Los autores actualizaron las estadísticas durante la celebración del torneo con el fin de dotar de más peso a los partidos más recientes de forma que se podía tener en consideración la forma actual de los jugadores. Consideraron que en un partido a 3 sets se juegan unos 165 puntos de media. Además, aunque asumieron una probabilidad constante en el caso del servidor durante todo el partido, indicaron que esta podría alterarse de ser necesario. Dichos autores concluyeron que era posible manipular las estadísticas publicadas sobre los jugadores para predecir el resultado de un partido y que siempre que se enfrentaban dos jugadores con un servicio dominante pero con restos débiles, siempre hay una probabilidad de que el partido llegue al $5^{\circ}$ set o se alargue.

Barnett y colaboradores (2004) demostraron como los tenistas pueden optimizar sus posibilidades de ganar un partido utilizando estrategias que les permitan usar sus recursos energéticos. Esto puede realizarse aumentando el esfuerzo en ciertos puntos, juegos y sets durante un partido, o aumentando o disminuyendo el esfuerzo en relación a una media total. Los resultados de su investigación demostraron que aumentar el esfuerzo en cualquier juego antes de alcanzar el "deuce" tiene el mismo efecto sobre las posibilidades de victoria del jugador en ese juego. Esforzarse más en los puntos importantes y menos en los puntos menos importantes hará que el jugador aumente la posibilidad de ganar el juego. En este estudio llegaron a la conclusión de que, un jugador que en un partido de 5 sets domine por 2 sets a 0 ó 2-1 y que esté perdiendo en el tercer o cuarto set, probablemente debería no esforzarse tanto en ese set que está perdiendo y guardar su energía para intentar ganar el set siguiente.

Con respecto a la cantidad de esfuerzo en los juegos de un set, estos autores concluyeron que si un jugador tiene mejor servicio que otro, es mejor que se esfuerce más al principio del set si está 
sacando, pero menos si el que saca es su adversario debido a que el mejor sacador tiene más posibilidades de ganar los puntos al servicio que el peor sacador.

En su investigación, Pollard y colegas (2006) concluyeron que en los datos obtenidos en partidos del Grand Slam, los mejores jugadores tenían mayores posibilidades de jugar mejor cuando iban perdiendo el partido que los peores jugadores.

Otro estudio centrado en el análisis diferencial entre ganadores y perdedores en los torneos de Wimbledon y de Roland Garros fue el de Katić y colaboradores (2011), donde los resultados de este trabajo mostraron diferencias en todas las variables analizadas excepto en las relacionadas con la velocidad del servicio. Las conclusiones de su trabajo mostraron que en Wimbledon las variables que se mostraron más determinantes para el resultado final fueron el porcentaje de puntos ganados al resto, el porcentaje de puntos ganados con el primer y segundo servicio, el porcentaje de puntos de break conseguidos, el porcentaje de puntos ganados en la red, el número de servicios directos y golpes ganadores y el número de errores no forzados. En Roland Garros, las variables determinantes fueron las mismas que en Wimbledon excepto el número de servicios directos, el porcentaje de puntos ganados en la red y el porcentaje de errores no forzados, donde no se encontraron diferencias entre los ganadores y perdedores.

Martínez-Gallego y colaboradores (2013) también realizaron una comparativa entre ganadores y perdedores en partidos disputados sobre superficie rápida en pista cubierta, en este caso analizaron la relación existente entre la posición en la pista y el número de puntos ganadores y errores. Según los resultados obtenidos, los perdedores cometieron un mayor porcentaje de errores no forzados que los ganadores, y estos últimos consiguieron un mayor porcentaje de puntos ganadores y errores forzados que el oponente. Además, se mostró como los ganadores consiguieron más puntos ganadores y 
forzaron al rival a cometer errores cuando se encontraban en zonas ofensivas de la pista, y por el contrario, los perdedores cometieron un mayor número de errores no forzados cuando se encontraban en dichas zonas ofensivas. (Martínez-Gallego, 2015).

\section{Diferencias entre el tenis masculino y femenino}

Magnus y Klaassen (2007) observaron que, en los datos analizados de Wimbledon, los hombres sacan tres veces más "aces" que las mujeres pero cometen el mismo número de doble faltas. El porcentaje de aces se define como la ratio entre número de aces (de 1er o $2^{\circ}$ servicio) y el número de puntos en los que se sacó, en lugar del número de servicios.

En este mismo estudio se llegó a la conclusión de que existe una diferencia entre la calidad del servicio entre hombres y mujeres. En los hombres, la diferencia de calidad del primer servicio entre jugadores que eran o no sembrados estaba determinada por el porcentaje de puntos que se ganaban con el primer servicio, mientras que en el caso de las mujeres la diferencia de calidad estaba determinada fundamentalmente por el porcentaje de primeros servicios que entran. En este mismo estudio también se llegó a la conclusión de que la diferencia entre jugadores cabezas de serie y no cabezas de serie era mayor en el tenis femenino que en el masculino.

O'Donoghue y Brown (2008) intentaron comprobar el principio de que en el tenis de alto rendimiento el sacador gana más puntos que el restador, concretamente si se relaciona con el número de aces y puntos de servicio o si esta ventaja también se da en peloteos de 3 ó más golpes. Los datos de partidos del Grand Slam permitieron concluir que en hombres la ventaja se mantiene en puntos de 3 ó 4 golpes para el primer saque pero no para el segundo. En el $2^{\circ}$ servicio, los hombres perdían la ventaja cuando el peloteo llegaba 
al tercer golpe. Por el contrario, en el tenis femenino, la ventaja del servicio se perdía tras los dos primeros golpes del peloteo tras el primer servicio, y no se observó ventaja significativa tras el $2^{\circ}$ servicio. El ler servicio en el tenis masculino en los Grand Slams da una ventaja al sacador de forma que el $62.4 \%$ de los puntos de 3 a 4 golpes los ganan, lo cual es significantemente mayor que el $49.7 \%$ de los puntos ganados si el peloteo dura 5 ó más golpes.

Filipcic, Filipcic y Berendijas (2009) realizaron una comparación por sexos y también entre ganadores y perdedores en la competición de Roland Garros. Las conclusiones del estudio apuntan que en ambos sexos hay indicadores de rendimiento que diferencian a ganadores de perdedores, como por ejemplo el menor número de dobles faltas, de errores no forzados así como un mayor porcentaje de puntos ganados con primer y segundo servicio.

\section{Superficie y estrategia}

Takahashi y colaboradores (2006) utilizaron un sistema informático para clarificar las características de la táctica utilizada en diferentes superficies de torneos del Grand Slam. Tomaron datos de la duración de los golpes, puntos y el tiempo entre puntos. Se anotó el golpe final de cada punto, el resultado de cada punto y la duración de cada golpe. El tiempo entre el impacto del servicio y el impacto del resto determinó la duración del servicio. El tiempo de duración de los golpes de fondo se calculó como el tiempo de impacto de un jugador y el impacto de otro. Un test de chi-cuadrado se utilizó para comparar la distribución de los golpes finales en cada superficie. Los resultados mostraron que el porcentaje de golpes de fondo fue alto en tierra mientras que los porcentajes de golpes de ataque y de defensa eran altos en hierba. Además, el ratio de servicios era alto en cancha dura. La duración del primer servicio 
fue de 0.91 segundos en tierra, 0.71 en hierba y 0.73 en pista rápida. La duración de los golpes de fondo fue de 1.36 segundos en tierra, 1.34 en hierba y 1.35 en rápida. La diferencia de duración fue de un 2/100 de segundo.

Takahashi y colegas (2009) utilizaron un notebook para estudiar la duración de los golpes y analizar los factores temporales en partidos de individuales de Grand Slam (Roland Garros, Wimbledon y el Abierto de EEUU) de forma que se comparó el efecto de la superficie de la pista sobre factores temporales. Se concluyó que la duración del primer y $2^{\circ}$ servicio era mayor en Roland Garros y que los tenistas necesitaban una estrategia diferente entre este y el resto de los torneos. La duración de los golpes de fondo no mostró diferencias significativas entre los tres torneos pues los golpes de fondo y los peloteos tenían ritmos simulares en los tres.

\section{Hipótesis sobre táctica y estrategia en el tenis}

Magnus y Klaassen (2007) afirmaron que al principio del último set la probabilidad de victoria de hombres que no eran cabezas de serie respecto a los cabezas de serie era mayor que las mujeres en la misma situación. También concluyeron que si hay una correlación entre quién ganará el último set con respecto al que ha ganado el set anterior, la misma será más negativa que positiva. Igualmente observaron que tras un break, el jugador que ganó el juego es más probable que gane el siguiente. Por tanto, no es cierto que tras un break el ganador se esfuerza menos y el perdedor más, sino que ocurre lo contrario, el ganador tiene más confianza y el perdedor se desanima.

Igualmente, estos autores concluyeron que los juegos en un partido de tenis no parecen ser independientes, sino que se produce una correlación positiva entre ellos: si ganas el juego anterior, ganarás el actual. Es lo que denominan una "tendencia ganadora" que se 
da más entre jugadores que son cabezas de serie que entre los que no son cabezas de serie, lo cual indica que los cabezas de serie son no sólo técnicamente sino también psicológicamente más fuertes que los que no son cabezas de serie.

O'Donoghue y Brown (2009) estudiaron secuencias de puntos de servicio en varios partidos de tenis de individuales para analizar si existía el "momentum" en tenis. Los resultados demostraron que ni los test de chi cuadrado ni los de Wald Wolfowitz encontraron más secuencias de puntos con el mismo resultado que se hubieran encontrado al azar ( $p>0.05$ ). Los autores concluyeron que no existe momentum en las secuencias de puntos analizadas en el tenis de individuales y que la creencia de jugadores, entrenadores, comentaristas y espectadores de que hay momentum en el tenis es una percepción errónea.

Loffing y colegas (2009) estudiaron si los jugadores zurdos tenían ventaja al sacar, especialmente cuando lo hacían contra jugadores diestros en el cuadro de ventaja. Para ello utilizaron la tecnología de seguimiento automático de la pelota "Hawk-Eye". Los autores concluyeron que los saques de jugadores diestros y zurdos diferían significativamente en cuanto a la colocación en el cuadro de servicio del contrario tanto en el primer y segundo servicio como en el ángulo lateral de vuelo de la pelota. Por lo que recomendaron que los restadores tuvieran en cuenta distintas probabilidades al esperar el servicio. Al mismo tiempo, también debían ajustar el movimiento de su resto al tipo de efecto del servicio. Además, los análisis revelaron que el saque de los zurdos no es similar al de los diestros sino significativamente diferente. Debido a la menor familiaridad con el estilo de juego de los zurdos, los resultados sugieren que estos aspectos técnico-tácticos pueden provocar la ventaja de los zurdos al sacar.

Utilizando la metodología de los sistemas dinámicos, Palut y Zannone (2005) investigaron la conducta colectiva de los tenistas 
durante un partido, considerando que en el tenis los desplazamientos de un lado a otro partiendo de una posición de referencia localizada en el centro de la línea de fondo son similares a los de un péndulo $\mathrm{y}$, por tanto, los movimientos recíprocos de ambos jugadores establecen una relación informativa. Por tanto, el desplazamiento de ambos jugadores puede analizarse como un sistema formado por dos péndulos no-lineales. En este sistema se ha observado que la fase relativa puede ser una medida de la sincronización temporal entre los péndulos. Los autores hipotetizaron que la fase relativa es una variable colectiva relevante que puede caracterizar el movimiento relativo de los dos tenistas. Los resultados revelaron la existencia de dos patrones estables de sincronización, in-fase y anti-fase, según los jugadores se iban moviendo en la misma o en direcciones opuestas, por lo que se confirmó la hipótesis.

\section{Importancia de los puntos, juegos y sets en tenis}

El tenis es un juego en el que la estructura del sistema de puntuación es jerárquica (Barnett et al., 2004). Como consecuencia del sistema de puntuación en el tenis, es posible que un jugador gane el partido ganando simplemente mucho menos que la mitad de los puntos jugados (Ferris, 2003). Esto plantea la pregunta de si algunos puntos, juegos o sets son más importantes que otros y si los jugadores deben distribuir su energía de acuerdo a esto.

Magnus y Klaassen (2007) observaron que la diferencia entre un jugador cabeza de serie y un no cabeza de serie decrece a lo largo del partido y, especialmente, en el set final. Pese a esto la probabilidad de que el jugador cabeza de serie gane el set final no decrece, sino que aumenta. Lo anterior se explica por la existencia de puntos más importantes que otros y el hecho de que los jugadores cabeza de serie jueguen mejor esos puntos que los no cabeza 
de serie o, igualmente, que los no cabeza de serie jueguen peor los puntos importantes. Estos mismos autores confirmaron que los verdaderos campeones juegan mejor los puntos importantes que el resto de los tenistas.

Bartlett y colegas (2004) afirmaron que, obviamente, en un partido al mejor 3 sets, el set más importante es el $3^{\circ}$, y que no es relevante si el esfuerzo mayor se hace en el primer set, y luego se reduce el esfuerzo en el $2^{\circ}$, o se reduce el esfuerzo en el $1^{\circ}$ y se aumenta en el $2^{\circ}$. Además, concluyeron que los mejores jugadores pueden variar sus esfuerzos más que los jugadores de menor nivel. Con respecto a la importancia de los puntos en un juego, estos autores afirman que un jugador puede aumentar su esfuerzo en cualquier punto del juego siempre que este esfuerzo se lleve a cabo antes de alcanzar la puntuación de "deuce". En el caso de un jugador que tiene el porcentaje medio de puntos ganados con su servicio, el punto de 30-40 es el más importante en un juego, junto con el "deuce", mientras que el menos importante es el de 40-0.

Magnus y Klaassen (2007) estudiaron la importancia del séptimo juego en un partido y llegaron a la conclusión de que estadísticamente no era relevante.

\section{Propuestas de entrenamiento de la táctica en el tenis}

Una vez expuestos los modelos de análisis de la táctica y las últimas investigaciones al respecto de la misma, consideramos importante en este capítulo presentar algunas orientaciones respecto al diseño de situaciones de entrenamiento de la táctica que podemos tener en cuenta, atendiendo a los conceptos vistos anteriormente. De hecho, al respecto de este tópico de entrenamiento de la táctica y, concretamente, de la toma de decisiones en el tenis, se han realizado diferentes aproximaciones en los últimos años tal y 
como señalan Carvalho y colaboradores (2011), que han pasado por los programas de entrenamiento perceptivo-cognitivo a través del desarrollo de estrategias visuales, donde la mejoría de las habilidades decisionales y anticipatorias de los deportistas se realizaba optimizando su capacidad percetiva, entendida como un proceso anterior a la decisión; por los programas de entrenamiento basado en la comprensión previa a la acción, asociando que el desarrollo de conocimiento está relacionado con los procesos cognitivos, de forma que no sólo está relacionado con el proceso decisional, sino que interviene también en otros procesos como la percepción; y, por último, por la propuesta de un nuevo programa de entrenamiento de la toma de decisiones a partir de las situaciones problemas que aporten información suficiente para que el tenista descubra las acciones más relevantes, utilizando los condicionantes o "constreñimientos" (Davids et al., 2008). Este último modelo, basado en los paradigmas cognitivos pero centrados en la teoría de los sistemas dinámicos y la perspectiva ecológica, aportando tareas que generen variabilidad e incertidumbre en su ejecución y que permitan realizar las adaptaciones pertinentes para poder resolver la situación problema que se plantea, es en el que nos quedaremos para poder plantear algunas tareas que, desde nuestro punto de vista, correspondan a lo que la realidad del juego de tenis compromete, una situación con incertidumbre, donde no es posible prever todo lo que puede suceder y que, por lo tanto, debemos preparar a nuestros jugadores para que sean capaces de tomar decisiones durante el juego adaptándose en todo momento a la situación que se presenta.

Los "constraints" en el diseño de las propuestas de entrenamiento, suelen referirse a tres aspectos, el entorno, la tarea y el deportista. La mayoría de ocasiones se desarrollarán basándonos en la tarea, ya que es la más sencilla a la hora de proponer las propuestas.

De esta forma los "constraints" centrados en el tenista incluirían aspectos tales como aspectos de la composición corporal (altura, 
peso, longitud de las extremidades), condición física (fuerza, velocidad, capacidad aeróbica, flexibilidad,..), habilidades psicológicas (concentración, confianza, control de las emociones, motivación,...), habilidades percetivas y de toma de decisión (reconocimiento de patrones de juego, anticipación ante las acciones del oponente,...) y aspectos personales (jugador más arriesgado o más conservador).

Respecto a los "constraints" centrados en el entorno incluirían aspectos tanto físicos como sociales del entorno. Dentro de los físicos podríamos hablar de la gravedad, la temperatura ambiente, la luz natura, el terreno o superficie de juego, el feedback recibido,..., y por lo que se refiere a las sociales podríamos hablar de las normas culturales, grupos de iguales, familia, ...

Por último, como señalábamos anteriormente, los más usuales y relacionados con el rendimiento son los "constraints" de la tarea, en donde se incluyen las reglas del deporte, equipamientos, número y disposición de los jugadores,...

En este sentido, las propuestas de práctica variable a través de manipulaciones de las condiciones de la tarea suponen una forma de entrenamiento con el objetivo de optimizar el rendimiento de nuestros jugadores y plantear situaciones de aprendizaje-entrenamiento de la táctica en entornos ecológicos. Debemos ser conscientes que este tipo de propuestas deben ser aplicadas de forma intermitente y con diferentes niveles de carga, adaptándonos siempre a los niveles de adaptación de nuestros jugadores a las tareas propuestas, de forma que sean un reto, pero que sea asumible, si bien es cierto, que necesitamos más investigación en este tópico para poder garantizar las bondades de esta propuesta frente al resto de opciones. En nuestro caso, y dado que se respetan las condiciones de incertidumbre que les exigirá la situación de competición a nuestros jugadores, recomendamos la utilización de las mismas como propuesta de trabajo para el entrenamiento de la táctica. 
Es cierto que con jugadores de alta competición, en numerosas ocasiones, y dependiendo también del jugador, se busca una "automatización de patrones tácticos, de forma que el jugador no tenga muchas opciones a la hora de resolver una situación problema que se le presenta tal y como señala Piles y Crespo (2012)

Sin embargo y pese a lo anteriormente señalado, debemos ser conscientes de que el jugador de alto rendimiento compite mucho más que entrena, encontrándose así sujeto a situaciones de un alto componente de variabilidad e incertidumbre durante gran parte del año, por lo que las situaciones de entrenamiento pueden tender a complementar una parte que quizás es más compleja de cubrir durante la competición como es la estabilidad de ciertas acciones técnicas o táticas. Sin embargo, con jugadores en formación, la situación puede ser muy diferente, por lo que las propuestas de entrenamiento deberían ponerles en situación de lo que se encontrarán posteriormente en un partido real. De ahí que deba ser el técnico quien decida el tipo de entrenamiento táctico a realizar, teniendo en cuenta las características del deportista, las necesidades del mismo y las exigencias de la competición.

Concluimos este apartado con algunos ejemplos de entrenamiento de la táctica en situaciones de práctica variable y aplicando los "constraints" sobre la tarea, con el objetivo de dejar explorar al jugador las posibles opciones y respuestas.

- Ejercicio 1: "Cruzados cambiados"

Objetivo: Trabajo de la consistencia y regularidad en el intercambio. Descripción: Jugar desde el fondo de la pista una situación de 1x1 en cruzado pero tenemos que golpear con el golpe contrario, es decir, en la situación de derecha contra derecha golpearemos de revés y viceversa en el lado contrario de la pista.

- Ejercicio 2: La autopista

Objetivo: Control y Concentración. 
Descripción: La pista de tenis estará limitada, de forma que sólo se hará un pasillo en el centro de la pista de dos metros, quedando la marca central en el centro. Se juega el punto debiendo golpear cada vez por un lado (derecha o revés).

- Ejercicio 3: Anticipación

Objetivo: Anticiparse a la acción del oponente.

Descripción: Situación de 1x1 donde uno de los jugadores estará sobre la línea de fondo y el otro juega libre desde el fondo de la pista. El objetivo será ganar el punto para evitar estar sobre la línea de fondo. El jugador que está pisando la línea no puede salir de ella para golpear por lo que deberá prever el golpe que va a realizar el adversario para moverse con antelación.

- Ejercicio 4: Defensa-Ataque

Objetivo: Mantener el rol asignado de defensa o ataque durante el punto.

Descripción: En situación de 1x1 se disputa el punto debiendo uno de los jugadores adoptar el rol defensivo, por lo que sus tiros serán con profundidad, altura y alejados de las líneas, evitando el margen de error. El otro jugador será ofensivo y deberá tirar más raso y potente, llevando la iniciativa en el punto. En el momento que una pelota no corresponde con el rol asignado se intercambian los papeles.

- Ejercicio 5: El cono prohibido

Objetivo: Resolución de problemas.

Descripción: Situación de 1x1 donde cada uno dispone de un cono que colocará en una de las 4 zonas de la pista (cuadro de servicio derecho o izquierdo, y cuadros de fondo tras la línea de servicio derecho e izquierdo). Este cono será la zona donde el adversario no podrá tirar la pelota porque entonces pierde el punto. Cada jugador no sólo evitará tirar la pelota al lugar donde está el cono, sino que buscará la forma de cómo su adversario puede tirar la pelota a la zona donde está el cono. 
- Ejercicio 6: El semáforo

Objetivo: Trabajo de toma de decisiones.

Descripción: Situación de 1x1 donde cada uno debe decir en voz alta, antes de golpear, el color que corresponda a la situación de juego en que se encuentra y, por lo tanto, asociado a la resolución que planteará. El color rojo corresponde a situaciones donde la respuesta será defensiva o neutra porque está en una situación de compromiso, el color naranja corresponde a una situación en donde se puede intentar tomar la iniciativa y forzar al adversario, mientras que el color verde supone una situación en la que se puede intentar ganar el punto y desbordar al adversario.

- Ejercicio 7: Doble red en profundidad

Objetivo: Resolución de problemas.

Descripción: Situación de 1x1 donde la red estará, además de la red habitual, sobre la línea de servicio de uno de los dos campos. De forma que para uno de los jugadores, su acción de jugar profundo está condicionado por la doble red y que le obligará a golpear con mayor profundidad. Cuando el técnico lo indique cambiarán el rol o podrán tirar una dentro de las dos redes y otra más allá de las dos redes.

- Ejercio 8: Doble red en altura

Objetivo: Toma de decisiones.

Descripción: Situación de 1x1 donde uno de los jugadores debe jugar por debajo de la doble red y el otro por encima hasta que el profesor indique lo contrario. cada uno dispone de un cono que colocará en una de las 4 zonas de la pista (cuadro de servicio derecho o izquierdo, y cuadros de fondo tras la línea de servicio derecho e izquierdo). Este cono será la zona donde el adversario no podrá tirar la pelota porque entonces pierde el punto. Cada jugador no sólo evitará tirar la pelota al lugar donde está el cono, sino que buscará la 
forma de cómo su adversario puede tirar la pelota a la zona donde está el cono.

- Ejercicio 9: El imitador

Objetivo: Resolución de problemas.

Descripción: Situación de 1x1 donde uno de los jugadores adopta el rol de líder y por lo tanto el patrón táctico que desarrolle será imitado por su adversario (defensivo, ofensivo, neutro, juego en profundidad, en anchura,...). Cada punto será un jugador el que marque lo que se debe hacer y el siguiente punto será reproducido por el otro jugador.

- Ejercicio 10: Los patrones de juego

Objetivo: Toma de decisión.

Descripción: Situación de 1x1 donde se disputa un Match Tie-Break y los dos jugadores deben tener un patrón de juego concreto que se administra o seleccionan ellos antes de comenzar. Tras jugar el set, se les pregunta cómo se ha desarrollado su patrón predefinido y si lo han podido llevar a término, y, en su caso, si han tenido que variar algo para conseguir ser efectivos. Posteriormente se les deja jugar de forma libre y al finalizar se les pregunta de nuevo por su patrón y por el del adversario, contrastando las respuestas para ver si han detectado las intenciones del oponente.

\section{Bibliografia}

Almajai, I., Kittler, J., de Campos, T., Christmas, W., Yan, F., Windridge, D., \& Khan, A. (2010). Ball event recognition using HMM for automatic tennis annotation, Proceedings of International Conference on Image Processing. Hong Kong.

Anguera, M.T. (1990). Metodología observacional. In J. Arnau, M.T. Anguera, J. Gómez (Eds.), Metodología de la Investigación en Ciencias del Comportamiento. Secretariado de Publicaciones de la Universidad de Murcia.

Anguera, M.T., Blanco, A., Losada, J.L., \& Hernández, A. (2000). La metodología observacional en el deporte: conceptos básicos. Lecturas: Educación Física y Deportes, 5, 24. 
Antoun, R. (2007). Using variety from the baseline in women's tennis. ITF Coaching and Sport Science Review, 43, 2-3.

Araújo, D. (2005). O Contexto da Decisão. A Acção Táctica no Desporto. Lisboa: Visão e Contextos.

Araujo, D., Davids, K., \& Hristovski, R. (2006). The ecological dynamics of decision making in sport. Psychology of Sport and Exercise, 7, 653-676.

Barnett, T., Brown, A., \& Clarke, S.R. (2004). Optimal use of tennis resources, Proceedings of the 7th Conference on Mathematics, Computers in Sport (pp. 5765). RH Morton and S. Ganesalingam.

Barnett, T., \& Clarke, S.R. (2005). Combining player statistics to predict outcomes of tennis matches. IMA Journal of Management Mathematics, 16, 113-120.

Barnett, T., Meyer, D., \& Pollard, G. (2008). Applying match statistics to increase serving performance. Medicine and Science in Tennis, 13, 24-27.

Bedford, A., Barnett, T., Pollard, G.H., \& Pollard, G.N. (2010). How the interpretation of match statistics affects player performance. Journal of Medicine and Science in Tennis, 15, 23-27.

Carvalho, J., Araújo, D., García, L., \& Iglesias, D. (2011). El entrenamiento de la toma de decisiones en el tenis: ¿qué fundamentos científicos se pueden aplicar en los programas de entrenamiento? Revista de Psicología del Deporte, 20, 767-783.

Christmas, W.J., Kostin, A., Yan, F., Kolonias, I., \& Kittler, J. (2005). A system for the automatic annotation of tennis matches, Fourth International Workshop on Content-Based Multimedia Indexing.

Crespo, M., \& Reid, M. (2002). Introducción a la táctica moderna. ITF Coaching and Sport Science Review, 27, 2.

Crespo, M., \& Sanz, D. (2011). Aportaciones de la investigación al tenis. Asignatura del Master de Enseñanza, Entrenamiento y Gestión del Tenis VIU-RFET. Documento Inédito.

Davids, K., Button, C., \& Bennett, S. (2008). Dynamics of skill acquisition: a constraints-led approach. Champaign, IL: Human Kinetics.

Filipcic, A., Zecic, M., Reid, M., Crespo, M., Panjan, A., \& Nejc, S. (2015). Differences in performance indicators of elite tennis players in the period 1991-2010. Journal of Physical Education and Sport, 15, 671-677.

Gale, D. (1971). Optimal strategy for serving in tennis. Mathematics Magazine, 44, 197-199.

Garay, O. (2003). Observación y Análisis de la Acción de Juego en el Tenis de Dobles (Doctoral Thesis). Universidad País Vasco, Bilbau.

George, S.L. (1973). Optimal strategy in tennis: a simple probabilistic model. Applied Statistics, 22, 97-104.

Gillet, E., Leroy, D., Thouvarecq, R., \& Stein, J.F. (2009). A Notational Analysis of Elite Tennis Serve and Serve-Return Strategies. Journal of Strength and Conditioning Research, 23, 532-539.

Gorospe, G. (1999). Observación y Análisis en el Tenis de Individuales. Aportaciones del análisis secuencial y de las coordenadas polares (Doctoral Thesis). Universidad País Vasco, Bilbau. 
Gorospe, G., Hernández, A., Anguera, M.T., \& Martínez de Santos, R. (2005). Desarrollo y optimización de una herramienta observacional en el tenis de individuales. Psicothema, 17, 123-127.

Hizan, H., Whipp, P., \& Reid, M. (2011). Comparison of serve and serve return statistics of high performance male and female tennis players from different age-groups. International Journal of Performance Analysis in Sport, 11, 365-375.

Katić, R., Milat, S., Zagorac, N., \& Đurović, N. (2011). Impact of game elements on tennis match outcome in Wimbledon and Roland Garros 2009. Collegium Antropologicum, 35, 341-346.

Klaassen, F.J.G.M., \& Magnus, J.R. (2003). Forecasting the winner of a tennis match. European Journal of Operational Research, 148, 257-267.

Martens, S., \& Maes, C. (2005). Periodisation for professional female tennis players. ITF Coaching and Sport Science Review, 36, 13-15.

Martínez-Gallego, R. (2015). Análisis notacional en el tenis. Revista Electrónica E-Coach de la RFET, 24, 4-9.

Norman, J.M. (1985). Dynamic programming in tennis-when to use a fast serve. Journal of Operational Research Society, 36, 75-77.

Norton, P., \& Clarke, S.R. (2002). Serving up some Grand Slam Statistics. In H. Morton (Eds.) Proceedings of the Sixth Australian Conference on Mathematics and Computers in Sport (pp. 202-209). Bond University, Queensland.

Over, S., \& O'donoghue, P. (2008). Whats the point tennis analysis and why. ITF Coaching and Sport Science Review, 45, 19-21.

O'Donoghue, P. (2001). The Most Important Points in Grand Slam Singles Tennis. Research Quarterly for Exercise and Sport, 72, 125-131.

O'Donoghue, P., \& Brown, E. (2009). Sequences of service points and the misperception of momentum in elite tennis. International Journal of Performance Analysis in Sport, 9, 113-127.

Palut, Y., \& Zanone, P.G. (2005). A dynamical analysis of tennis: Concepts and data. Journal of Sports Sciences, 23, 1021-1032.

Piles, J., \& Crespo, M. (2012). Tactics for elite level men's tennis - Part 1. ITF Coaching and Sport Science Review, 56, 9-10.

Pollard, G.N., Pollard, G.H., Barnett, T., \& Zeleznikow, J. (2009). Applying tennis match statistics to increase serving performance during a match in progress. Journal of Medicine and Science in Tennis, 14, 16-19.

Pollard, G.N., Pollard. G.H., Barnett, T., \& Zeleznikow, J. (2010). Applying strategies to the tennis challenge system. Medicine and Science in Tennis, 15, 12-15.

Reid, M. (2011). Moneyball for tennis, ITF Worlwide Coaches Conference. Egypt.

Rodriguez, C. (2012). El tenis femenino, pasado, presente y ifuturo? ITF Coaching and Sport Science Review, 56, 21-22.

Reid, M., McMurtrie, D., \& Crespo, M. (2010). The relationship between match statistics and top 100 ranking in professional men's tennis. International Journal of Performance in Analysis in Sport, 10, 131-138.

Sanz-Rivas, D., \& Terroba, A. (2012). Aplicación de las nuevas tecnologías al análisis de la táctica en el tenis. ITF Coaching and Sport Science Review, 56, 23-25. 
Sanz, D. (2015). Evaluación y Análisis del Rendimiento. Asignatura del Master de Enseñanza, Entrenamiento y Gestión del Tenis MEDAC-RFET-UI1. Documento Inédito.

Sanz, D., Fuentes, J.P., Del Villar, F., \& Julián, J.A. (2004). El Tenis en la Escuela. Barcelona: Paidotribo.

Schutz, R.W. (1970). A mathematical model for evaluating scoring systems with specific reference to tennis. Research Quarterly for Exercise and Sport, 41, 552-561.

Takahashi, H., Wada, T., Maeda, A., Kodama, M., Nishizono, H., \& Kurata, H. (2006). The relationship between court surface and tactics in tennis using a computerized scorebook. International Journal of Performance Analysis in Sport, 6, 15-25.

Takahashi, H., Wada, T., Maeda, A., Kodama, M., \& Nishizono, H. (2009). An analysis of time factors in elite male tennis players using the computerised scorebook for tennis. International Journal of Performance Analysis in Sport, 9, 314-319.

Terroba, A., Kosters, W.A., \& Vis, J.K. (2010). Tactical Analysis Modeling through Data Mining: Pattern Discovery in Racket Sports, International Conference on Knowledge Discovery and Information Retrieval (KDIR 2010). Valencia.

Tiley, C. (2002). A winning game plan for all court players. ITF Coaching and Sport Science Review, 27, 7-9.

Vis, J.K., Kosters, W.A., \& Terroba, A. (2010). Tennis Patterns: Player, Match and Beyond. 22nd Benelux Conference on Artificial Intelligence (BNAIC 2010). Luxembourg.

Van Aken, I. (2002). Tactics specific to the female game. ITF Coaching and Sport Science Review, 27, 13-14. 